\title{
Connection between response parameter and anomaly coefficient in two dimensional anomalous fluid
}

\author{
Bibhas Ranjan Majhi \\ Racah Institute of Physics, Hebrew University of Jerusalem, \\ Givat Ram, Jerusalem 91904, Israel \\ E-mail: bibhas.majhi@mail.huji.ac.il
}

ABSTRACT: In $(1+1)$ dimensional hydrodynamics in presence of the gravitational anomalies, the constitutive relations for the stress tensor contain the response parameters $\bar{C}_{1}$, $\bar{C}_{2}$ and the gravitation anomaly coefficients $c_{g}, c_{w}$. Here it is shown that they are related by the two relations $\bar{C}_{1}=4 \pi^{2} c_{w}$ and $\bar{C}_{2}=8 \pi^{2} c_{g}$. This agrees with the earlier findings. I argue that the Israel-Hartle-Hawking vacuum is the natural boundary condition which leads to such relation. Finally, the possible physical implications are discussed.

Keywords: 2D Gravity, Anomalies in Field and String Theories, Field Theories in Lower Dimensions

ARXiv EPRINT: 1401.1074 


\section{Contents}

1 Introduction $\quad 1$

2 Two dimensional anomalous fluid: a brief summary 1

3 Relation between response parameters and anomaly coefficients $\quad 2$

4 Conclusions 4

\section{Introduction}

The relevant equation of motion for the hydrodynamics are the certain conservation equations which are supplemented by the constitutive relations - the energy-momentum tensor and the current expressed in terms of the fluid variables [1]. The role of anomalies in fluid dynamics has been a central attention in recent years [2]-[7]. The central purpose, so far, is to find the constitutive relations by solving the anomaly expressions. It turns out that the these relations can be expressed in terms of fluid variables, like temperature, chemical potential etc. under the static background. This is possible because the fluid variables at an arbitrary spacetime point are related to their equilibrium values through the background metric coefficients which is known as the Tolman relation [8].

The constitutive relations for the stress tensor in presence of the gravitational anomalies usually contain certain factors, like response parameters and the coefficients of the anomaly expressions (for details, see [3]-[7]). In this paper, I will show that there exists two simple relations between them. It agrees with the earlier findings [4]. Contrary to the existing work [4], the present analysis will be simple and will provide more physical insight in this paradigm. It will be shown that the imposition of the Israel-Hartle-Hawking vacuum condition on the two dimensional anomalous stress tensor yields the required result.

Here the detail derivation of anomalous constitutive relation will not be given, because it has been already done earlier $[6,7]$. To achieve the goal, I will start from the anomalous constitutive relations which are derived in [7] and then proceed further. At the end, the physical implications will be discussed.

The organization of the paper is as follows. In section 2 , the form of the anomalous constitutive relations for the energy-momentum tensor will be given. Then I shall find the relations between the response parameters and the anomaly coefficients in section 3 . Final section will be devoted for the conclusions and physical interpretations.

\section{Two dimensional anomalous fluid: a brief summary}

In this section, the explicit form of the constitutive relations for the energy-momentum tensor in presence of the gravitational anomalies in $(1+1)$ dimensions will be given. The 
background is chosen to be static. Here, I will not give the detail derivation of these relations since it can be followed from [7].

It is well known that the anomalies are the intrinsic properties of field theory. In $(1+1)$ the anomaly expressions are given by [4] (see also [9-13]):

$$
\nabla_{b} T^{a b}=c_{g} \bar{\epsilon}^{a b} \nabla_{b} R ; \quad T_{a}^{a(g)}=c_{w} R
$$

where $c_{g}$ and $c_{w}$ are two normalization constants and $R$ is the two dimensional Ricci scalar. Following the steps employed in $[6,7]$, it can be shown that in the comoving frame under the static background:

$$
d s^{2}=-e^{2 \sigma(r)} d t^{2}+g_{11} d r^{2},
$$

the components of stress tensor, obtained from the solutions of the anomaly expressions eq. (2.1), can be casted in the constitutive relation for stress tensor of a fluid:

$$
\begin{aligned}
T_{a b}= & {\left[2 c_{w}\left(u^{c} \nabla^{d}-u^{d} \nabla^{c}\right) \nabla_{c} u_{d}+2 \bar{C}_{1} T^{2}\right] u_{a} u_{b} } \\
& -\left[2 c_{g}\left(u^{c} \nabla^{d}-u^{d} \nabla^{c}\right) \nabla_{c} u_{d}+\bar{C}_{2} T^{2}\right]\left(u_{a} \tilde{u}_{b}+\tilde{u}_{a} u_{b}\right) \\
& +\left[\bar{C}_{1} T^{2}-c_{w}\left(u^{c} \nabla^{d} \nabla_{d} u_{c}\right)\right] g_{a b} .
\end{aligned}
$$

Here $u^{a}$ is the fluid velocity, $\tilde{u}_{a}=\bar{\epsilon}_{a b} u^{b}$ and $T$ is related to the equilibrium temperature $T_{0}$ by the relation $T=e^{-\sigma} T_{0} . u^{a}$ is chosen such that in the comoving frame it satisfies the timelike condition $g_{a b} u^{a} u^{b}=-1$. For a detail analysis and the meaning of the symbols, see [7]. The similar relation was also obtained by derivative expansion approach in [4]. The response parameters $\bar{C}_{1}$ and $\bar{C}_{2}$ are related to the equilibrium temperature $T_{0}$ of the fluid as,

$$
\bar{C}_{1}=\left(C_{u u}+C_{v v}\right) T_{0}^{-2} ; \quad \bar{C}_{2}=\left(C_{u u}-C_{v v}\right) T_{0}^{-2}
$$

where $C_{u u}$ and $C_{v v}$ are the integration constants appearing in the solution of eq. (2.1).

Now it must be noted that in the above constitutive relation eq. (2.3) there exists four parameters: two response parameters $\bar{C}_{1}$ and $\bar{C}_{2}$ and two anomaly coefficients $c_{g}$ and $c_{w}$. In the next section I will provide a relation between them.

\section{Relation between response parameters and anomaly coefficients}

In the derivative expansion approach [4], the authors of the paper showed that the response parameters $\bar{C}_{1}$ and $\bar{C}_{2}$ are proportional to the normalization constants $c_{w}$ and $c_{g}$ appearing in the anomaly expressions, respectively. The exact connection is given by,

$$
\bar{C}_{1}=4 \pi^{2} c_{w} ; \quad \bar{C}_{2}=8 \pi^{2} c_{g} .
$$

(See, eq. (4.15a) and eq. (4.15b) of [4] with the identifications $\bar{C}_{1}=p_{0}$ and $\bar{C}_{2}=-\tilde{c}_{2 d}$ ). The evidence of this statement can be followed from earlier works in various subfield of physics [14]-[18]. The derivation in [4] was done by demanding that the thermal energymomentum tensor on the cone must vanishes in the Euclidean vacuum. The procedure is 
very much technically involved. Here, in this paper, a simple realization of this relation will be presented. I shall show that one can understand it by just fixing the integration constants $C_{u u}$ and $C_{v v}$ upon imposing a relevant boundary condition.

To proceed towards the main goal, let us first express the components of $T_{a b}$ in terms of the metric coefficients under the background (2.2) in null coordinates $(u, v)$. From eq. (2.3), these are given by,

$$
\begin{aligned}
& T_{u u}=\frac{2 c_{g}+c_{w}}{4} \frac{e^{2 \sigma}}{g_{11}^{2}}\left(2 \sigma^{\prime \prime} g_{11}-\sigma^{\prime} g_{11}^{\prime}\right)+C_{u u} ; \\
& T_{v v}=-\frac{2 c_{g}-c_{w}}{4} \frac{e^{2 \sigma}}{g_{11}^{2}}\left(2 \sigma^{\prime \prime} g_{11}-\sigma^{\prime} g_{11}^{\prime}\right)+C_{v v} ; \\
& T_{u v}=-\frac{c_{w}}{4} e^{2 \sigma} R .
\end{aligned}
$$

Here the prime denotes the derivative with respect to " $r$ " coordinate. Now to determine the integration constants $C_{u u}$ and $C_{v v}$ we need to impose a suitable boundary condition. In literature there exists three types of vacua, corresponding to the different boundary conditions [19]. Among them, here we shall show that Israel-Hartle-Hawking vacuum condition is the relevant one to achieve the required relation. This vacuum is defined by the fact that the stress tensor in Kruskal coordinates corresponding to the both outgoing and ingoing modes must be regular near the horizon. Hence $T_{u u} \rightarrow 0$ and $T_{v v} \rightarrow 0$ near the horizon. To impose these conditions, let us consider that the metric (2.2) is the solution of the Einstein equation and since the metric is static, the Killing horizon and the event horizon must coincide [20]. Therefore, in the present case, $\left.e^{2 \sigma}\right|_{r_{0}}=0=1 /\left.g_{11}\right|_{r_{0}}$ where $r_{0}$ is the position of the horizon. Now denoting $e^{2 \sigma(r)} \equiv f(r)$ and $1 / g_{11}(r) \equiv g(r)$, we expand them the near the horizon as

$$
f(r)=f^{\prime}\left(r_{0}\right)\left(r-r_{0}\right)+\ldots ; \quad g(r)=g^{\prime}\left(r_{0}\right)\left(r-r_{0}\right)+\ldots
$$

Substituting these in the $u u$ component of $T_{a b}$ (see eq. (3.2)) and then taking the limit $r \rightarrow r_{0}$, we obtain expression as

$$
T_{u u}=-\frac{2 c_{g}+c_{w}}{4} \frac{f^{\prime}\left(r_{0}\right) g^{\prime}\left(r_{0}\right)}{2}+C_{u u} .
$$

Now since near horizon this must vanishes, we get

$$
C_{u u}=\frac{\left(2 c_{g}+c_{w}\right) \kappa^{2}}{2}=2 \pi^{2}\left(2 c_{g}+c_{w}\right) T_{0}^{2}
$$

where the surface gravity $\kappa=\sqrt{f^{\prime}\left(r_{0}\right) g^{\prime}\left(r_{0}\right)} / 2$ and the equilibrium temperature $T_{0}=\kappa / 2 \pi$. Similarly, imposition of the same boundary condition on eq. (3.3) leads to

$$
C_{v v}=-2 \pi^{2}\left(2 c_{g}-c_{w}\right) T_{0}^{2}
$$

Substitution of these in eq. (2.4) lead to both the two required relations in eq. (3.1).

Before I conclude, let me make some comments on the relations of eq. (3.1) and the way these have been derived here. In the context of $(1+1)$ dimensional conformal field theory 
(CFT) and Cardy formula, there exits similar results like eq. (3.1). It is well known that the Cardy formula relates the pressure of the CFT with the left and right handed central charges by the relation $P=2 \pi T^{2}\left(c_{L}+c_{R}\right) / 24=4 \pi^{2} c_{w} T^{2}$ where $c_{w}$ is the same trace anomaly coefficient as given in eq. (2.1) (see [21, 22], for details). Furthermore, the arguments employed here to derive the relations are similar to those used for deriving the Cardy formula. Here we imposed the Israel-Hartle-Hawking vacuum condition which for Killing horizons implies that the stress-tensor must be regular on the corresponding Euclidean cigar. Moreover, it is possible to derive the relations by using the similar formalism as that of the derivation of the Cardy formula. This has been shown in [4]. On the other hand if one calculates the $T_{r}^{r}$ component, for the present case, which is the pressure, then it turns out to be $T_{r}^{r}=4 \pi^{2} c_{w} T^{2}+$ (higher derivative terms). This is exactly the expression, mentioned above, obtained by Cardy formula. In addition, it can be shown that $T_{t}^{r}=8 \pi^{2} T^{2} c_{g}$ and $-T_{t}^{t}=4 \pi^{2} c_{w} T^{2}$ upto some higher derivative terms. All these suggest that the method, employed here, is similar to that for deriving the Cardy formula.

\section{Conclusions}

In this paper, a simple derivation of the relations in (3.1) has been given in the context of two dimensional anomalous hydrodynamics. I showed that these are coming from the fixing of the integration constants, appearing in the solutions of the gravitational anomaly equations, by imposing the Israel-Hartle-Hawking vacuum condition. Such an analysis, so far I know, does not exist in literature. It clearly revels the importance of the vacuum condition in the anomalous hydrodynamics. I believe that the present analysis might shed some light towards this paradigm.

The physical significance is as follows. It is well known that in the thermodynamics of gravity, the main macroscopic entities are temperature, entropy, free energy, etc. which are all observer dependent quantities. For instance, a freely falling observer through the black hole horizon does not associate these on the horizon while the thermodynamical parameters are well defined with respect to the outside static observer. Therefore, one can argue that the degrees of freedom responsible for them are not absolute [23]-[25]. Similarly, as we have seen in the present analysis, the relevant observer is one which corresponds to the Israel-Hartle-Hawking vacuum. Hence the underlying microscopic theory for the anomalous fluid dynamics may have observer dependent notion. Finally, the present analysis is general enough to include higher dimensional theories. Furthermore, to understand more about the significance of the vacuum it would be interesting to study the gravitation anomalies in higher dimensions. This I leave for the future.

\section{Acknowledgments}

I thank Rabin Banerjee and S. Jain for several useful discussions. I also like to thank the referee for various important comments. The research of the author is supported by a Lady Davis Fellowship at Hebrew University, by the I-CORE Program of the Planning and Budgeting Committee and the Israel Science Foundation (grant No. 1937/12), as well as by the Israel Science Foundation personal grant No. 24/12. 
Open Access. This article is distributed under the terms of the Creative Commons Attribution License (CC-BY 4.0), which permits any use, distribution and reproduction in any medium, provided the original author(s) and source are credited.

\section{References}

[1] L.D. Landau and E.M. Lifshitz, Course of Theoretical Physics. Vol. 6: Fluid Mechanics, Elsevier, Oxford U.K. (1987).

[2] S. Dubovsky, L. Hui and A. Nicolis, Effective field theory for hydrodynamics: Wess-Zumino term and anomalies in two spacetime dimensions, arXiv:1107.0732 [INSPIRE].

[3] M. Valle, Hydrodynamics in $1+1$ dimensions with gravitational anomalies, JHEP 08 (2012) 113 [arXiv:1206.1538] [INSPIRE].

[4] K. Jensen, R. Loganayagam and A. Yarom, Thermodynamics, gravitational anomalies and cones, JHEP 02 (2013) 088 [arXiv:1207.5824] [INSPIRE].

[5] S. Jain and T. Sharma, Anomalous charged fluids in $1+1 d$ from equilibrium partition function, JHEP 01 (2013) 039 [arXiv: 1203.5308] [INSPIRE].

[6] R. Banerjee, Exact results in two dimensional chiral hydrodynamics with diffeomorphism and conformal anomalies, arXiv:1303.5593 [INSPIRE].

[7] R. Banerjee, P. Chakraborty, S. Dey, B.R. Majhi and A.K. Mitra, Two dimensional hydrodynamics with gauge and gravitational anomalies, arXiv:1307.1313 [INSPIRE].

[8] R.C. Tolman, Relativity, Thermodynamics and Cosmology, Dover Publication, New York U.S.A. (1987), pg. 318.

[9] W.A. Bardeen and B. Zumino, Consistent and Covariant Anomalies in Gauge and Gravitational Theories, Nucl. Phys. B 244 (1984) 421 [InSPIRE].

[10] L. Álvarez-Gaumé and P.H. Ginsparg, The Structure of Gauge and Gravitational Anomalies, Annals Phys. 161 (1985) 423 [Erratum ibid. 171 (1986) 233] [INSPIRE].

[11] L. Álvarez-Gaumé and E. Witten, Gravitational anomalies, Nucl. Phys. B 234 (1984) 269 [INSPIRE].

[12] R. Banerjee and S. Kulkarni, Hawking Radiation, Covariant Boundary Conditions and Vacuum States, Phys. Rev. D 79 (2009) 084035 [arXiv: 0810.5683] [INSPIRE].

[13] R. Banerjee and B.R. Majhi, Connecting anomaly and tunneling methods for Hawking effect through chirality, Phys. Rev. D 79 (2009) 064024 [arXiv:0812.0497] [INSPIRE].

[14] G. Volovik and A. Vilenkin, Macroscopic parity violating effects and He-3-A, Phys. Rev. D 62 (2000) 025014 [hep-ph/9905460] [INSPIRE].

[15] A. Cappelli, M. Huerta and G.R. Zemba, Thermal transport in chiral conformal theories and hierarchical quantum Hall states, Nucl. Phys. B 636 (2002) 568 [cond-mat/0111437] [INSPIRE].

[16] M. Stone, Gravitational Anomalies and Thermal Hall effect in Topological Insulators, Phys. Rev. B 85 (2012) 184503 [arXiv:1201.4095] [INSPIRE].

[17] K. Landsteiner, E. Megias and F. Pena-Benitez, Gravitational Anomaly and Transport, Phys. Rev. Lett. 107 (2011) 021601 [arXiv:1103.5006] [INSPIRE]. 
[18] P. Kraus and F. Larsen, Holographic gravitational anomalies, JHEP 01 (2006) 022 [hep-th/0508218] [INSPIRE].

[19] R. Balbinot, A. Fabbri and I.L. Shapiro, Vacuum polarization in Schwarzschild space-time by anomaly induced effective actions, Nucl. Phys. B 559 (1999) 301 [hep-th/9904162] [INSPIRE].

[20] B. Carter, Black Hole Equilibrium States, in Black Holes, C. DeWitt and B.S. DeWitt eds., Gordon and Breach, New York U.S.A. (1973), pg. 57.

[21] H. Bloete, J.L. Cardy and M. Nightingale, Conformal Invariance, the Central Charge and Universal Finite Size Amplitudes at Criticality, Phys. Rev. Lett. 56 (1986) 742 [INSPIRE].

[22] I. Affleck, Universal Term in the Free Energy at a Critical Point and the Conformal Anomaly, Phys. Rev. Lett. 56 (1986) 746 [InSPIRE].

[23] B.R. Majhi and T. Padmanabhan, Noether current from the surface term of gravitational action, Virasoro algebra and horizon entropy, Phys. Rev. D 86 (2012) 101501 [arXiv: 1204.1422] [INSPIRE].

[24] B.R. Majhi, Noether current of the surface term of Einstein-Hilbert action, Virasoro algebra and entropy, Adv. High Energy Phys. 2013 (2013) 386342 [arXiv:1210.6736] [INSPIRE].

[25] B.R. Majhi and T. Padmanabhan, Thermality and Heat Content of horizons from infinitesimal coordinate transformations, Eur. Phys. J. C $\mathbf{7 3}$ (2013) 2651 [arXiv:1302.1206] [INSPIRE]. 